\title{
Obtenção de ferritas de cálcio dopadas com cromo visando aplicação em esmaltes transparentes brilhante e mate
}

\author{
Obtaining calcium ferrites doped with chromium \\ aims application in transparent \\ and matte glazes
}

Gerbeson Carlos Batista Dantas ${ }^{1}$, Jairo Luís dos Santos Dutra ${ }^{1}$, Patrícia Mendonça Pimentel ${ }^{1}$, Sâmea Valensca Alves Barros ${ }^{1}$

\begin{abstract}
${ }^{1}$ Universidade Federal Rural do Semi-Árido Campus Angicos 59515-000, Angicos, RN, Brasil. e-mail: gerbeson_dantas@hotmail.com, jairoluisdsd@gmail.com, pimentelmp@ufersa.edu.br, sameavalensca@ufersa.edu.br
\end{abstract}

\section{RESUMO}

Este trabalho tem como objetivo obter óxidos com estrutura tipo espinélio de composição $\mathrm{CaFe}_{1,8} \mathrm{Cr}_{0,2} \mathrm{O}_{4}$ pelo método gelatina, visando aplicação em esmaltes transparentes brilhantes e mate. Os pós precursores, resultantes do processo de síntese, foram calcinados a $600{ }^{\circ} \mathrm{C}$ e a $800{ }^{\circ} \mathrm{C}$ para a obtenção da fase espinélio. Os pós foram caracterizados por espectroscopia na região do infravermelho, difração de raios-x, seguido por refinamento Rietveld, espectroscopia de reflectância difusa UV-visível e colorimetria CIE-L*a*b*. Em seguida, os pós foram misturados aos esmaltes transparentes brilhante e mate na proporção de $2 \%$ em peso, revestido substratos cerâmicos e, posteriormente, sinterizados a $1100{ }^{\circ} \mathrm{C}$. De acordo com os difratogramas de raios-x, os pós possuíram estrutura espinélio ortorrômbica. As ferritas de cálcio dopadas com cromo apresentaram coloração alaranjada, sendo mais escura nos pós calcinados a temperaturas mais altas. Os resultados da esmaltação revelaram que houve compatibilidade entre o pigmento e o esmalte brilhante. Já nos esmaltes mate, houve variação abrupta da cor. $\mathrm{O}$ ataque por ácido clorídrico mostrou que os esmaltes preparados são altamente resistentes ao ataque químico. Por fim, este trabalho conclui que a rota gelatina foi eficiente em sintetizar óxidos com estrutura espinélio monofásicos em baixas temperaturas de síntese e que os pigmentos obtidos são viáveis para aplicação industrial.

Palavras-chave: Gelatina, Espinélios, Propriedades ópticas, Ferritas, Dopagem.

\begin{abstract}
This work aims to obtain oxides with a spinel type structure of composition $\mathrm{CaFe}_{1,8} \mathrm{Cr}_{0,2} \mathrm{O}_{4}$ by the gelatin method, aiming application in transparent and matte glazes glazes. The precursor powders, resulting from the synthesis process, were calcined at $600^{\circ} \mathrm{C}$ and $800^{\circ} \mathrm{C}$ to obtain the spinel phase. The powders were characterized by infrared spectroscopy, X-ray diffraction, followed by Rietveld refinement, diffuse UV-visible reflectance spectroscopy and CIE-L*a*b* colorimetry. The powders were then incorporated into the bright and matte transparent glazes in the proportion of $2 \%$ by weight, coated ceramic substrates and then sintered at $1100{ }^{\circ} \mathrm{C}$. According to the X-ray diffractograms, the powders had an orthorhombic spinel structure. The calcium ferrites doped with chromium presented orange coloration, being darker in the calcined powders at higher temperatures. The glaze coating results showed that there was compatibility between pigment and glaze brigth. We already mate glaze, there was abrupt variation of color. The hydrochloric acid attack showed that the prepared glazes are highly resistant to chemical attack. Finally, this work concludes that the gelatin route was efficient in synthesizing oxides with a single-phase spinel structure at low synthesis temperatures and that the obtained pigments are viable for industrial application.
\end{abstract}

Keywords: Gelatin, Spinel, Optical Properties, Ferrites, Doping. 


\section{INTRODUÇÃO}

A estrutura espinélio possui formulação geral $\mathrm{AB}_{2} \mathrm{O}_{4}$. $\mathrm{O}$ sítio A é composto por elementos do grupo dos metais com estado de oxidação II, enquanto o sítio B representa um íon metálico trivalente. A estrutura ideal do espinélio possui simetria cúbica de face centrada e 96 interstícios catiônicos, sendo 64 interstícios tetraédricos e 32 octaédricos. Entretanto, somente 24 interstícios são ocupados, sendo 8 tetraédricos e 16 octaédricos [1-2]. Há também a estrutura espinélio invertida, de modo que a quantidade de sítios tetraédricos e octaédricos se invertem [1]. Nesse sentido, a estrutura espinélio tem atraído cada vez mais atenção dos pesquisadores devido sua versatilidade de arranjo dos cátions nos sítios (normal ou invertida) bem como, devido a possibilidade de incorporação de dopagens substitucionais [3].

Um dos compostos com estrutura espinélio que tem sido frequentemente estudado é o grupo das ferritas. Em geral, estes compostos resultam em pigmentos de cor avermelhada, bastante empregada na indústria cerâmica. Contudo, com a crescente demanda por pigmentos e as novas exigências tecnológicas, estudos de abrangência internacional tem sido realizado no intuito de sintetizar pigmentos com cores diversas e, principalmente, com boa qualidade [3-4]. Um pigmento de boa qualidade deve apresentar estabilidade química e térmica, não geração gases e flocos na superfície do esmalte [5]. Frequentemente, essas propriedades são atribuídas à estrutura e elementos químicos que compõem os pigmentos. Entretanto, sabe-se que a rota de síntese exerce influência substancial nas propriedades finais, bem como na homogeneidade destas propriedades [6].

A principal rota de síntese empregada na indústria é a rota cerâmica [7]. Este método tem produzido pigmentos com propriedades heterogêneas, depreciando o valor agregado e a vida útil da esmaltação. Rotas alternativas vêm sendo utilizadas para sintetizar as ferritas, tais como, Pechine, combustão, coprecipitação e reação no estado sólido. No entanto, estas rotas estão associadas à elevada perda de material ou ainda, utilização de temperaturas de calcinação muito elevadas, inviabilizando sua reprodução em escala industrial.

Nos últimos anos, um método que vem chamando atenção é o método gelatina. Nesse método de síntese, a gelatina comestível é utilizada como agente polimerizante para a obtenção de óxidos com alta pureza e baixo custo. A gelatina é uma proteína composta por aminoácidos essenciais, substituindo os produtos tóxicos usando em outras rotas [8-9].

Dentre as principais vantagens deste método está a alta homogeneidade do material preparado, a obtenção de partículas nanométricas, além da simplicidade, do baixo custo envolvido e não geração de resíduos tóxicos $[4,6]$. Somando-se a isso, outra vantagem do método é a facilidade na obtenção da gelatina, visto que esta é um polímero natural, obtido pela hidrólise das fibras de colágeno, normalmente utilizando como matérias-primas as peles de origem animal e tecnologia simples e dominada pela indústria e, por essa razão, é capaz de ser produzida em larga escala [10-11]. Não obstante, a produção mundial de gelatina alcançou a marca recorde de 412.700 toneladas em 2015, com perspectiva de crescimento cada vez mais acentuada [12].

Nesse contexto, diante da escassez de pesquisas de conteúdo nacional no desenvolvimento de pigmentos e rotas de síntese, este trabalho tem como objetivo obter óxidos com estrutura tipo espinélio de composição $\mathrm{CaFe}_{1,8} \mathrm{Cr}_{0,2} \mathrm{O}_{4}$ pelo método gelatina, avaliando as propriedades microestruturais e ópticas dos pós produzidos, visando aplicação em esmaltes transparentes brilhantes e mate.

\section{MATERIAIS E MÉTODOS}

\subsection{Materiais}

Os materiais usados na síntese dos pós foram: nitrato de ferro (III) nonahidratado $\mathrm{Fe}\left(\mathrm{NO}_{3}\right)_{3} \cdot 9 \mathrm{H}_{2} \mathrm{O}$ (SigmaAldrich, 98,9\%), nitrato de cálcio tetrahidratado $\mathrm{Ca}\left(\mathrm{NO}_{3}\right)_{3} \cdot 4 \mathrm{H}_{2} \mathrm{O}$ (Vetec, $99,8 \%$ ) e nitrato de cromo hexahidratado $\mathrm{Cr}\left(\mathrm{NO}_{3}\right)_{3} \cdot 6 \mathrm{H}_{2} \mathrm{O}$ (Sigma-Aldrich, 99,8\%). A gelatina foi cedida pela empresa GELITA. Os esmaltes transparentes brilhantes e mate foram cedidos pela empresa Vittra. As placas cerâmicas foram fornecidas pela empresa BQMIL. 


\subsection{Método}

Os pós foram preparados utilizando a rota gelatina, conforme descrito por outros autores [4,6,13-15]. Inicialmente, a gelatina foi misturada à água deionizada, sob agitação, até sua dissolução completa. Em seguida, foram adicionados os nitratos metálicos obedecendo a relação estequiométrica 1:1 entre gelatina/íons metálicos. Então, a mistura permaneceu sob agitação e temperatura de $70{ }^{\circ} \mathrm{C}$ até a formação de um gel, levado a mufla por $2 \mathrm{~h}$ para eliminação da matéria orgânica inserida no processo de síntese. Em seguida, os pós precursores foram calcinados nas temperaturas previamente estabelecidas $\left(600^{\circ} \mathrm{C}\right.$ e $\left.800^{\circ} \mathrm{C}\right)$ por $4 \mathrm{~h}$ para obtenção da fase espinélio.

\subsection{Preparações das placas cerâmicas}

O esmalte foi preparado a partir de uma mistura de $2 \%$ em peso de pigmentos e $98 \%$ de esmaltes transparente brilhante (TB) e transparente mate (TM). Em seguida, os esmaltes foram utilizados para revestir o substrato cerâmico. As placas cerâmicas foram levadas a mufla por $15 \mathrm{~min}$ a $1100{ }^{\circ} \mathrm{C}$ para sinterização.

\subsection{Ataque químico}

Inicialmente, as cerâmicas preparadas com os esmaltes TB e TM foram imersas em uma solução aquosa contendo $15 \%(\mathrm{~V} / \mathrm{V})$ de $\mathrm{HCl}$ e mantidas imersa por $1 \mathrm{~h}$. Em seguida, foram lavadas com água deionizada até $\mathrm{pH}$ neutro, secos e novamente submetidos a análise da cor.

\subsection{Caracterização}

Após a obtenção dos pós, o material foi caracterizado por meio das técnicas: difração de raios-x com refinamento Rietveld por meio do equipamento modelo XRD-6000 do difratômetro Shimadzu, operando com radiação $\mathrm{CuK} \alpha$, ângulo de $2 \theta$ variando de $10^{\circ}$ a $80^{\circ}$; espectrometria na região do infravermelho utilizando o espectrofotômetro da marca Shimadzu; colorimetria por intermédio do colorímetro Gretag Macbeth color-eye 2180 e espectrometria na região do UV-Visível usando o espectrofotômetro UV-Visible Shimadzu, com reflectância acessória UV-2550 com um comprimento de onda na região de 200-900 nanômetros. A composição química dos esmaltes foi obtida por meio da espectroscopia de fluorescência de raios X utilizando o espectrômetro de fluorescência de raios-X por energia dispersiva (EDX), da marca Shimadzu, modelo EDX-720. As cerâmicas foram caracterizadas por colorimetria CIE-L*a*b*. As cerâmicas submetidas ao ataque químico foram submetidas a CIE-L*a*b*.

\section{RESULTADOS E DISCUSSÃO}

A Figura 1 evidencia os espectros vibracionais de transmissão na região do infravermelho (2000 $\mathrm{cm}^{-1} \mathrm{a}$ $400 \mathrm{~cm}^{-1}$ ) para os pós de $\mathrm{CaFe}_{1,8} \mathrm{Cr}_{0,2} \mathrm{O}_{4}$. De acordo com os estudos de Reddy e Frost (2005), os óxidos com

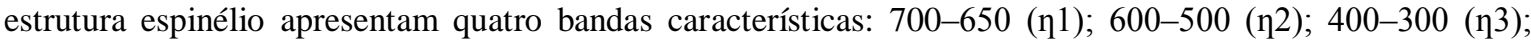
200-150cm-1 (n4). Essas bandas são características dos metais de transição com estado de oxidação II e III que, especificamente neste estudo são os íons $\mathrm{Fe}^{3+}$ e o $\mathrm{Cr}^{3+}$. Nesse sentido, observa-se na Figura 1, as bandas

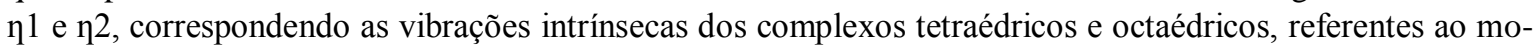
vimento de estiramento característico da estrutura espinélio [16-17]. 


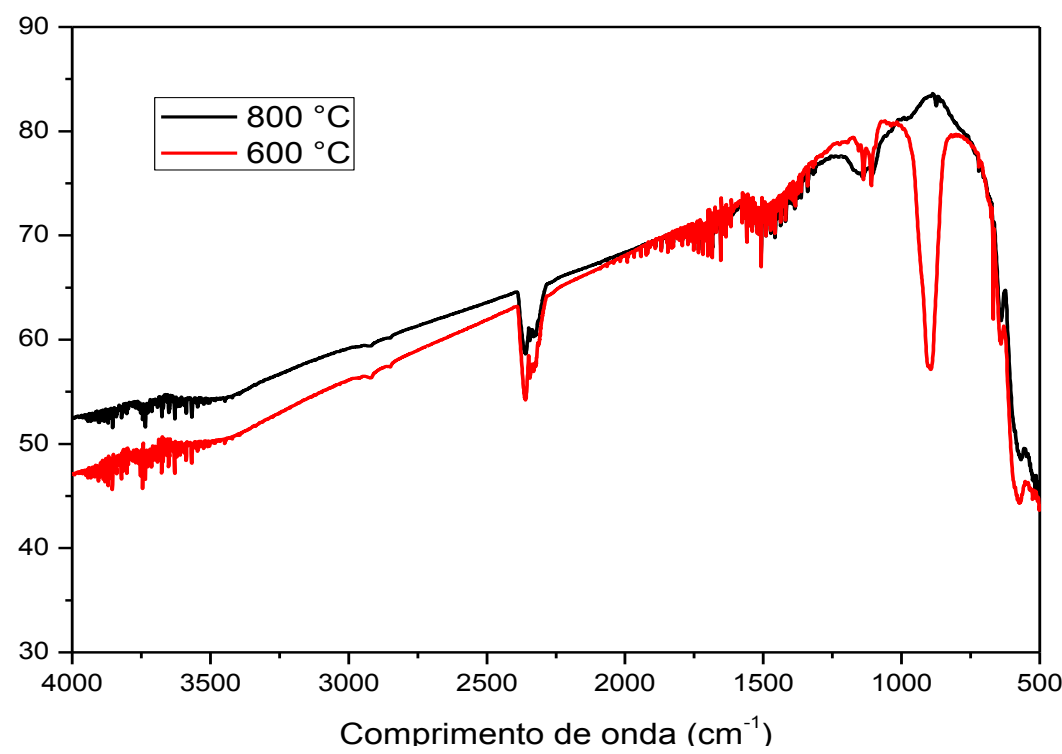

Figura 1: Espectros de transmissão de infravermelho das amostras de $\mathrm{CaFe}_{1,8} \mathrm{Cr}_{0,2} \mathrm{O}_{4}$

A Figura 2 apresenta os difratogramas de raios-x dos pós de ferritas de cálcio dopadas com cromo nas duas temperaturas de calcinação. A identificação das fases deu-se por meio do Refinamento Rietveld [18], utilizando o software MAUD [19]. Os difratogramas revelaram que os pós sintetizados apresentaram estrutura cristalina tipo espinélio, com simetria ortorrômbica, conforme constatada pela carta ICSD 16695, correspondente a ferrita de cálcio sem dopagem. A quantidade de reflexões, combinada com a reflexão principal em $30^{\circ}<\mathrm{x}<35^{\circ}$ são característicos da estrutura espinélio, corroborando os resultados do infravermelho. $\mathrm{O}$ estreitamento da base da reflexão principal evidencia o aumento da cristalinidade dos pós calcinados nas mais altas temperaturas. Observa-se também que o cromo incorporou completamente na rede cristalina, uma vez que a carta utilizada é correspondente a ferrita de cálcio sem dopagem $\left(\mathrm{CaFe}_{2} \mathrm{O}_{4}\right)$. Os pós sintetizados pela rota da gelatina foram monofásicos em ambas as temperaturas de calcinação, mostrando-se vantajoso para obtenção de ferritas de cálcio. O interesse por pós monofásicos ocorre pela necessidade dos pigmentos apresentarem propriedades homogêneas, agregando valor tecnológico e econômico, de modo a garantir qualidade, melhor desempenho e maior durabilidade do revestimento cerâmico aplicado, quando submetido aos esforços de abrasão, aos agentes químicos e solicitações físico-mecânicos.

Os parâmetros de refinamento estão ilustrados na Tabela 1. Com base nos resultados, o tamanho do cristalito aumento com a elevação da temperatura calcinação, o que é um resultado esperado, visto que a temperatura é a força impulsionadora de expansão do cristalito. O valor do refinamento foi aceitável, visto que foi inferior a 2 para ambos pós [9]. 


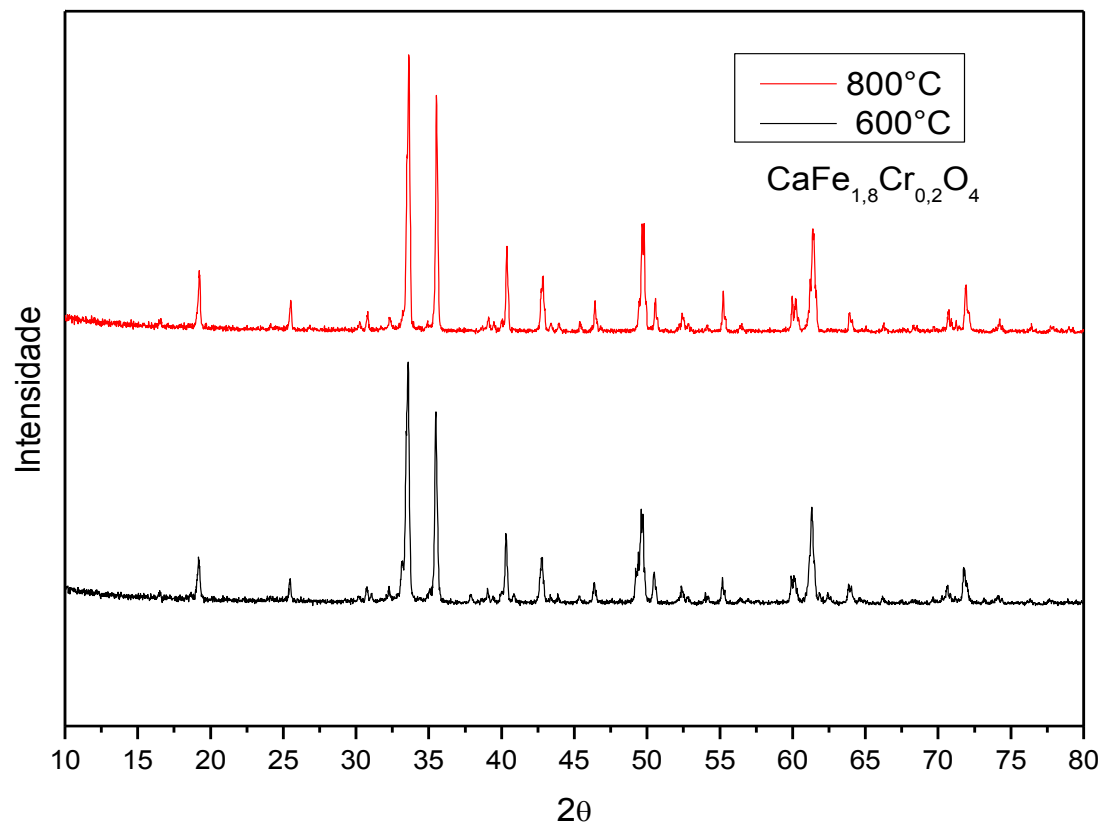

Figura 2: Difratogramas de raios-x dos das amostras de $\mathrm{CaFe}_{1,8} \mathrm{Cr}_{0,2} \mathrm{O}_{4}$.

Tabela 1: Parâmetros microestruturais das amostras de $\mathrm{CaFe}_{1,8} \mathrm{Cr}_{0,2} \mathrm{O}_{4}$.

\begin{tabular}{|c|c|c|c|c|c|c|c|}
\hline \multirow{2}{*}{ AMOSTRAS } & \multirow{2}{*}{$\begin{array}{c}\mathrm{T} \\
\left({ }^{\circ} \mathrm{C}\right)\end{array}$} & \multicolumn{4}{|c|}{ PARÂMETROS DE REDE (Å) } & \multirow{2}{*}{$\begin{array}{c}\text { TAMANHO DO } \\
\text { CRISTALITO (nm) }\end{array}$} & \multirow[t]{2}{*}{ SIG } \\
\hline & & $\mathbf{a}$ & b & c & $\mathbf{V}\left(\AA^{3}\right)$ & & \\
\hline \multirow{2}{*}{$\mathrm{CaFe}_{1,8} \mathrm{Cr}_{0,2} \mathrm{O}_{4}$} & 600 & 9,228 & 3,0199 & 10,7013 & 298,12 & 95 & 1,52 \\
\hline & 800 & 9,2122 & 3,0153 & 10,6928 & 297,01 & 460 & 1,89 \\
\hline
\end{tabular}

A Figura 3 e a Tabela 2 apresentam os espectros de reflectância difusa e os parâmetros colorimétricos das amostras de $\mathrm{CaFe}_{1,8} \mathrm{Cr}_{0,2} \mathrm{O}_{4}$, respectivamente. A partir do estudo da formação da cor, sabe-se que é um fenômeno que ocorre devido às transições $d$-d, proporcionado pelo não preenchimento total dos elétrons nos

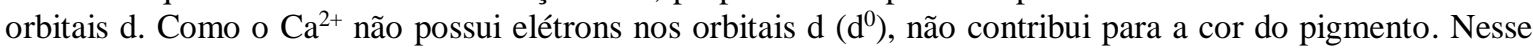
sentido, os íons $\mathrm{Cr}^{3+}$ e $\mathrm{Fe}^{3+}$ são os cromóforos do espinélio sintetizado, sendo o íon $\mathrm{Fe}^{3+}$ o principal contribuinte para a cor final, conforme evidenciado pela banda larga entre 450 e $700 \mathrm{~nm}$ no espectro de reflectância das amostras, sendo atribuída à transição ${ }^{6} \mathrm{~A}_{1 \mathrm{~g}}{ }^{4} \mathrm{~T}_{2 \mathrm{~g}}$, que é característica do íon $\mathrm{Fe}^{3+}$ em sítio octaédrico [2022]. Devido ao pequeno percentual de dopagem ( $10 \%$ de cromo) e a sobreposição das bandas do íon $\mathrm{Fe}^{3+}$, as transições do íon $\mathrm{Cr}^{3+}$ em sítio octaédrico não são observadas e, portanto, não exercem contribuição significativa na cor alaranjada do pigmento. Esses resultados são corroborados pelos parâmetros colorimétricos CIE$\mathrm{L}^{*} \mathrm{a} * \mathrm{~b} *$ da Tabela 2. Além disso, de acordo com estes parâmetros, os pós calcinados nas temperaturas em $1000{ }^{\circ} \mathrm{C}$ são mais escuros. Esse comportamento é justificado pelo aumento do tamanho do cristalito (Tabela 1) que reduz a superfície de reflexão da luz do iluminante, resultando em um deslocamento da luminosidade $\mathrm{L}$ para o preto [6]. Observa-se também que os pós apresentaram $\Delta \mathrm{E}_{0}$ inferior a 10 , revelando a estabilidade térmica dos pós sintetizados pelo método gelatina. 

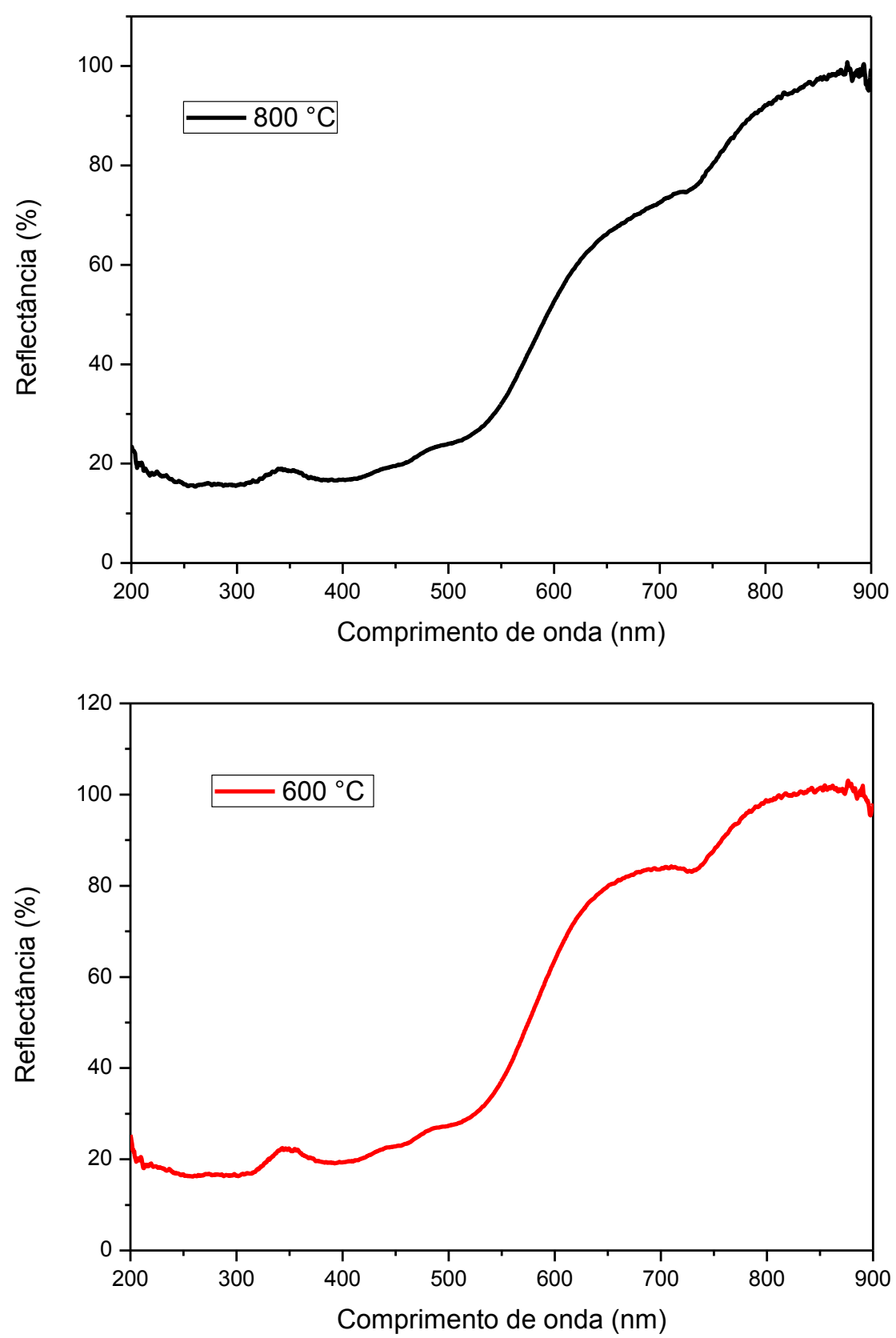

Figura 3: Espectros de reflectância difusa das amostras de $\mathrm{CaFe}_{1,8} \mathrm{Cr}_{0,2} \mathrm{O}_{4}$.

Tabela 2: Colorimetria CIE-L*a*b das amostras de $\mathrm{CaFe}_{1,8} \mathrm{Cr}_{0,2} \mathrm{O}_{4}$

\begin{tabular}{c|c|c|c|c|c}
\hline AMOSTRA & $\mathbf{T}\left({ }^{\circ} \mathbf{C}\right)$ & $\mathbf{L}$ & $\mathbf{a}^{*}$ & $\mathbf{b}^{*}$ & \multirow{2}{*}{$\mathbf{E}_{\mathbf{0}}$} \\
\hline $\mathrm{CaFe}_{1,8} \mathrm{Cr}_{0,2} \mathrm{O}_{4}$ & 600 & 45,5 & 12,9 & 26,0 & \multirow{2}{*}{9,53} \\
\hline $\mathrm{CaFe}_{1,8} \mathrm{Cr}_{0,2} \mathrm{O}_{4}$ & 800 & 39,9 & 5,5 & 23,6 & \\
\hline
\end{tabular}

A Tabela 3 evidencia as coordenadas colorimétricas dos esmaltes preparados a partir da incorporação dos pigmentos na matriz vítrea brilhante e mate. Observa-se que após a incorporação no esmalte TB, os parâmetros colorimétricos não sofreram deslocamento significativo, revelando que houve compatibilidade química entre os componentes do esmalte e do pigmento. A satisfatória estabilidade de cor do esmalte está apre- 
sentada na Tabela 3, que evidenciou $\Delta \mathrm{E}_{1}$ inferior a 20 . O bom desempenho da incorporação é explicado pela composição química do esmalte brilhante apresentado na Tabela 4. O elevado teor de sílica e alumina, combinado com o baixo teor de impurezas e de opacificante $(\mathrm{ZnO})$ resultaram na baixa reatividade do esmalte, preservando a cor dos pigmentos, conforme mostrado na Figura 4 [23-24].

A colorimetria dos esmaltes preparados com o esmalte TM pode ser visto na Tabela 3. Observa-se que apresentaram deslocamento significativo da cor na direção do preto, sinalizando que houve interação entre componentes químicos dos pigmentos e do esmalte mate. Provavelmente, o elevado teor de óxido de zinco $(\mathrm{ZnO}>10)$, combinando com elevado teor de óxido de cálcio resultou em um esmalte mais opaco e com menor capacidade de reflexão do esmalte, resultando em um $\Delta \mathrm{E}_{1}$ superior a $20 \mathrm{e}$, consequentemente, a coloração mais escura [23-24].

Tabela 3: Colorimetria CIE-L*a*b* das cerâmicas no esmalte brilhante e mate.

\begin{tabular}{|c|c|c|c|c|c|c|c|}
\hline AMOSTRA & $\mathbf{T}\left({ }^{\circ} \mathbf{C}\right)$ & ESMALTE & TIPO & $\mathbf{L}$ & $a^{*}$ & $\mathbf{b}^{*}$ & $\Delta \mathrm{E}_{1}$ \\
\hline $\mathrm{CaFe}_{1,8} \mathrm{Cr}_{0,2} \mathrm{O}_{4}$ & 600 & TB & Cerâmica & 55,6 & 7,3 & 32,1 & \multirow[t]{2}{*}{13,1} \\
\hline $\mathrm{CaFe}_{1,8} \mathrm{Cr}_{0,2} \mathrm{O}_{4}$ & 600 & - & Pigmento & 45,5 & 12,9 & 26,0 & \\
\hline $\mathrm{CaFe}_{1,8} \mathrm{Cr}_{0,2} \mathrm{O}_{4}$ & 800 & TB & Cerâmica & 52,1 & 12 & 33,7 & \multirow[t]{2}{*}{17,1} \\
\hline $\mathrm{CaFe}_{1,8} \mathrm{Cr}_{0,2} \mathrm{O}_{4}$ & 800 & - & Pigmento & 39,9 & 5,5 & 23,6 & \\
\hline $\mathrm{CaFe}_{1,8} \mathrm{Cr}_{0,2} \mathrm{O}_{4}$ & 600 & $\mathrm{TM}$ & Cerâmica & 19,8 & 2,7 & 11,4 & \multirow[t]{2}{*}{31,3} \\
\hline $\mathrm{CaFe}_{1,8} \mathrm{Cr}_{0,2} \mathrm{O}_{4}$ & 600 & - & Pigmento & 45,5 & 12,9 & 26 & \\
\hline $\mathrm{CaFe}_{1,8} \mathrm{Cr}_{0,2} \mathrm{O}_{4}$ & 800 & $\mathrm{TM}$ & Cerâmica & 18,9 & 3,5 & 11,6 & \multirow[t]{2}{*}{24,3} \\
\hline $\mathrm{CaFe}_{1,8} \mathrm{Cr}_{0,2} \mathrm{O}_{4}$ & 800 & - & Pigmento & 39,9 & 5,5 & 23,6 & \\
\hline
\end{tabular}

Tabela 4: Composição química do esmalte transparente brilhante e mate

\begin{tabular}{c|c|c|c|c|c|c|c|c}
\hline $\begin{array}{c}\text { COMPOSIÇÃO } \\
\text { QUÍMICA }\end{array}$ & $\begin{array}{c}\mathrm{SiO}_{2} \\
(\%)\end{array}$ & $\begin{array}{c}\mathrm{Al}_{2} \mathrm{O}_{3} \\
(\%)\end{array}$ & $\begin{array}{c}\mathrm{CaO} \\
(\%)\end{array}$ & $\begin{array}{c}\mathrm{K}_{2} \mathrm{O} \\
(\%)\end{array}$ & $\begin{array}{c}\mathrm{ZnO} \\
(\%)\end{array}$ & $\begin{array}{c}\mathrm{SO}_{3} \\
(\%)\end{array}$ & $\begin{array}{c}\mathrm{Fe}_{2} \mathrm{O}_{3} \\
(\%)\end{array}$ & $\begin{array}{c}\text { OUTROS } \\
(\%)\end{array}$ \\
\hline $\mathrm{TB}$ & 64,570 & 21,251 & 6,614 & 3,428 & 3,207 & 0,609 & 0,253 & 0,0599 \\
\hline $\mathrm{TM}$ & 34,861 & 9,515 & 15,376 & 2,130 & 34,685 & - & 0,370 & - \\
\hline
\end{tabular}

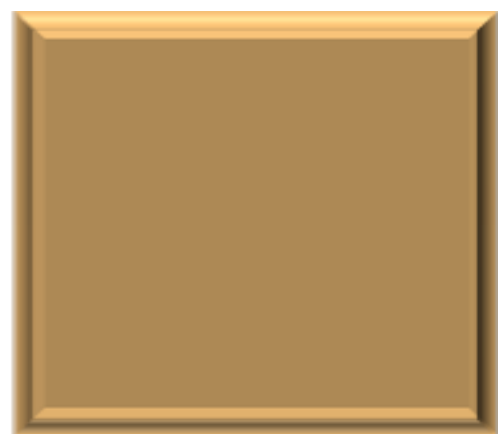

a)

c)

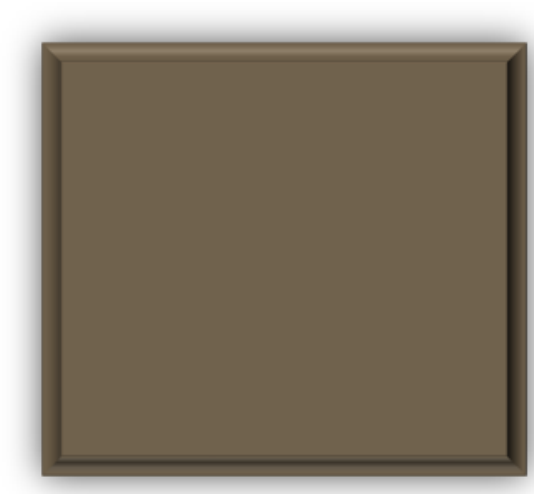


b)

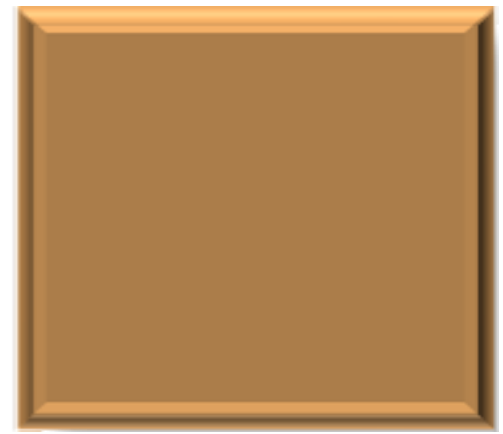

Figura 4: Imagens das cerâmicas obtidas a) $\mathrm{CaFe}_{1,8} \mathrm{Cr}_{0,2} \mathrm{O}_{4} 600^{\circ} \_\mathrm{TBc}$ e b) $\mathrm{CaFe}_{1,8} \mathrm{Cr}_{0,2} \mathrm{O}_{4} 800^{\circ} \mathrm{C}_{-} \mathrm{TB}$, c) $\mathrm{Ca}-$ $\mathrm{Fe}_{1,8} \mathrm{Cr}_{0,2} \mathrm{O}_{4} \_00{ }^{\circ} \mathrm{C}_{-} \mathrm{TM}$ e d) $\mathrm{CaFe}_{1,8} \mathrm{Cr}_{0,2} \mathrm{O}_{4} \_800^{\circ} \mathrm{C} \_\mathrm{TM}$.

Os resultados do teste de resistência ao ataque químico estão exibidos na Tabela 5. De acordo com os resultados, não houve alteração significativa da cor em todas as amostras, uma vez que a variação de cor foi inferior a 5, mostrando que os esmaltes preparados são capazes de resistir ao ataque químico de ácido clorídrico na concentração de $15 \%$ (v/v).

Tabela 5: Coordenadas CIE-L*a*b* das cerâmicas após ataque químico.

\begin{tabular}{|c|c|c|c|c|c|c|c|c|}
\hline AMOSTRA & $\mathrm{T}\left({ }^{\circ} \mathrm{C}\right)$ & ESMALTE & TIPO & $\mathbf{L}$ & $A^{*}$ & $\mathbf{B}^{*}$ & $\Delta \mathrm{E}_{2}$ & $\begin{array}{l}\text { VARIAÇÃO } \\
\text { DE COR }\end{array}$ \\
\hline \multirow{2}{*}{$\mathrm{CaFe}_{1,8} \mathrm{Cr}_{0,2} \mathrm{O}_{4}$} & \multirow[t]{2}{*}{600} & - & Pigmento & 45,5 & 12,9 & 26 & \multirow[t]{2}{*}{17,6} & \multirow[t]{2}{*}{4,52} \\
\hline & & $\mathrm{TB}$ & Cerâmica & 61,6 & 6,9 & 22,1 & & \\
\hline \multirow{2}{*}{$\mathrm{CaFe}_{1,8} \mathrm{Cr}_{0,2} \mathrm{O}_{4}$} & \multirow[t]{2}{*}{600} & - & Pigmento & 45,5 & 12,9 & 26 & \multirow[t]{2}{*}{35,7} & \multirow[t]{2}{*}{4,34} \\
\hline & & $\mathrm{TM}$ & Cerâmica & 15,8 & 2,3 & 9,4 & & \\
\hline \multirow{2}{*}{$\mathrm{CaFe}_{1,8} \mathrm{Cr}_{0,2} \mathrm{O}_{4}$} & \multirow[t]{2}{*}{800} & - & Pigmento & 39,9 & 5,5 & 23,6 & \multirow[t]{2}{*}{18,2} & \multirow[t]{2}{*}{1,10} \\
\hline & & TB & Cerâmica & 51,9 & 11,9 & 35,7 & & \\
\hline \multirow{2}{*}{$\mathrm{CaFe}_{1,8} \mathrm{Cr}_{0,2} \mathrm{O}_{4}$} & \multirow[t]{2}{*}{800} & - & Pigmento & 39,9 & 5,5 & 23,6 & \multirow[t]{2}{*}{28,4} & \multirow[t]{2}{*}{4,10} \\
\hline & & $\mathrm{TM}$ & Cerâmica & 15,9 & 3,1 & 8,6 & & \\
\hline
\end{tabular}

\section{CONCLUSÕES}

A rota de síntese gelatina revelou-se simples, de baixo custo e rápido no passo a formar óxidos de estrutura do tipo espinélio a baixas temperaturas, podendo ser usada para substituir outros métodos usais de síntese. Os difratogramas de raio- $\mathrm{x}$ mostraram que as ferritas de cálcio sintetizadas pelo método da gelatina exibiram estrutura do tipo espinélio com distorção ortorrômbica. A cor alaranjada deve-se predominantemente às bandas características do íon cromóforo $\mathrm{Fe}^{3+}$ em sítio octaédrico. Quanto ao estudo da interação entre os pigmentos e o esmalte transparente brilhante, a cor original do pigmento foi praticamente preservada, sinalizando que os componentes químicos do esmalte não reagiram com os pigmentos evidenciando, assim, estabilidade química do esmalte. Já com os esmaltes mate, a cor foi modificada para tons mais escuros, evidenciando uma possível interação entre compostos do esmalte mate e do pigmento. O ataque químico mostrou que os esmaltes preparados apresentam elevada resistência química. Por fim, conclui-se que os pigmentos preparados podem ser utilizados na indústria cerâmica. 


\section{AGRADECIMENTOS}

Os autores agradecem ao CNPQ pelo suporte financeiro à pesquisa, a empresa VITTRA pelo fornecimento dos esmaltes e a BQMIL pelo fornecimento dos substratos cerâmicos utilizados nesse trabalho.

\section{BIBLIOGRAFIA}

[1] SILVA, M.A.F.M., SOSMAN, L.P., YOKAICHIYA, F., et al., "Neutron Powder Diffraction Measurements of the Spinel $\mathrm{MgGa}_{2} \mathrm{O}_{4}: \mathrm{Cr}^{3+}$ - A Comparative Study between the High Flux Diffractometer D2B at the ILL and the High Resolution Powder Diffractometer Aurora at IPEN", Journal of Physics Conference Series, v. 340, pp. 12-41, 2012

[2] SOSMAN, L. P., FONSECA, R.J.M., TAVARES JR, A D et al., "Photoluminescence and optical absorption of $\mathrm{Cs}_{2} \mathrm{NaScF}_{6}: \mathrm{Cr}^{3+”}$, Journal of Fluorescence, v. 16, n.2, pp. 317-323, 2006.

[3] COSTA, A.F., PIMENTEL, P.M., MELO, D.M.A., et al., "Preparação de pigmentos de aluminatos de cobalto para aplicação em vidrados cerâmicos", Cerâmica, v. 62, pp. 179-185, 2016.

[4] DUTRA, J. L. S., DANTAS, G. C. B., PIMENTEL, P. M., et al., "Caracterização óptica e estrutural de ortoferritas de lantânio dopadas com cromo e alumínio", Cerâmica, v.64, pp.413-417, 2018.

[5] FURUKAWA, S., MASUI, T., IMANAKA, N. "Synthesis of new environment-friendly yellow pigments", J. Alloys Compd., v.418, pp. 255, 2006.

[6] PIMENTEL, P.M., LIMA, S.V.M., COSTA, A.F., et al., "Gelatin synthesis and color properties of (La, Pr, Nd) lanthanide aluminates”, Ceramics International, v.43, pp.6592-6596, 2017.

[7] ALBUQUERQE, G.B., BALLMANN, T.J.C., FOLGUERAS, M.V., et al., "Síntese de pigmento cerâmico verde com base na estrutura cristalina da wollastonita", Matéria, v.21, n.2, pp. 355-365, 2016.

[8] COSTA, A. F., PIMENTEL, P. M., MELO, D. M. A., et al., "Síntese e caracterização de espinélios a base de ferritas utilizando gelatina como agente direcionador", Cerâmica, v. 57, pp. 352-355, 2011.

[9] PIMENTEL, P.M., BARBOSA, K.M.B., GOMES, D.K.S., et al., "Optical and structural properties of lanthanum chromite synthesized by microwave assisted selfcombustion method", Materials Science Forum, v.881, pp.7-11, 2016.

[10] VIJAYARAGHAVAN, R., THOMPSON, B.C., MACFARLANE, D.R., et al., "Biocompatibility of choline salts as crosslinking agents for collagen based biomaterials", Chemical Communications, v.46, pp.294-296, 2010.

[11] DUCONSEILLE, A., GAILLARD, C., SANTÉ-LHOUTELLIER, V., et al., "Molecular and structural changes in gelatin evidenced by Raman microspectroscopy", Food Hydrocolloids, v. 77, pp. 777-786, 2018.

[12] GRAND VIEW RESEARCH. I Gelatin Market Analysis by Raw Material, Function, Application and Segment Forecasts to 2024, (June 2016 ed.)Grand View Research, Inc. 2016. Disponível em <https://www.grandviewresearch.com/industry-analysis/gelatin-market-analysis>. Acesso em: 17 de março de 2018 .

[13] OLIVEIRA, F.S., PIMENTEL, P.M., OLIVEIRA, R.M.P.B., et al., "Effect of lanthanum replacement by strontium in lanthanum nickelate crystals synthetized using gelatin as organic precursor", Materials Letter, v.64, pp.2700-2703, 2010.

[14] PIMENTEL, P.M., DUTRA, J. L. S., LIMA, A. C., et al., "Structural and magnetic characterization of LaFe1-xAlxO3 ( $x=0$ and 0.2) orthoferrites synthesized by gelatin method", Materials Science Forum, v. 899, pp. 227-231, 2017.

[15] COSTA, A.F., PIMENTEL, P.M., AQUINO, F.M., et al., "Microstructural and colour analysis of Cu$\mathrm{CrO}_{2}$ delafossite obtained by gelatin method", Materials Science Forum, v.798-799, pp.160-164, 2014.

[16] PATIL, R. S., KAKATKAR, S. V., SANKPAL, A. M., et al., "Infrared absorption spectra of Ti4+ and Zr4+ substituted Ni-Zn ferrites" Indian. J. Pure Appl. Phys., v.32, n. 193, 1994.

[17] REDDY, B. J., FROST, R. L. "Spectroscopic characterization of chromite from the moa-baracoa ophiolitic massif, Cuba", Spectrochimica Acta a, v.61, pp.1721, 2005.

[18] RIETVELD, H.M. "A profile refinement method for nuclear and magnetic structures", Journal of Applied Crystallography, v.2, pp.65-71, 1969.

[19] LUTTEROTTI, L. (2006). MAUD, Version 2.046, http://www.ing.unitn.it/wmaud/. 
[20] PAILHE, N., WATTIAUX, A., GAUDON, M., et al., "Correlation between structural features and visNIR spectra of $\alpha-\mathrm{Fe}_{2} \mathrm{O}_{3}$ hematite and $\mathrm{AFe}_{2} \mathrm{O}_{4}$ spinel oxides (A=Mg, Zn)", J. Solid State Chem., v.181, pp.1040-1047, 2008.

[21] KUMARI, L.S., RAO, P.P., REDDY, M.L. "Environment-friendly red pigments from $\mathrm{CeO}_{2}-\mathrm{Fe}_{2} \mathrm{O}_{3}-$ Pr6O11 solid solutions”, J. Alloy Compd., v.461, pp.509-515, 2008.

[22] MARANHA, F.G., FIUZA, T.E.R., SOUZA, E.C.F., et al., "Synthesis and characterization of pigments of the $\mathrm{LaAl}_{1-\mathrm{x}} \mathrm{FexO}_{3}$ system e Application in ceramic and polymer”, Dyes and Pigments, v.133, pp.304-310, 2016.

[23] AHMADI, S., AGHAEI, A., YEKTA, B.E, "Synthesis of $\mathrm{Y}(\mathrm{Al}, \mathrm{Cr}) \mathrm{O}_{3}$ red pigments by co-precipitation method and their interactions with glazes", Ceramics International, v.35, pp.3485-3488, 2009.

[24] PEREIRA, O.C., BERNARDIN, A.M. "Ceramic colorant from untreated iron ore residue", Journal of Hazardous Materials, v.233-234, pp.103-111, 2012.

\section{ORCID}

Gerbeson Carlos Batista Dantas Jairo Luís dos Santos Dutra Patrícia Mendonça Pimentel Sâmea Valensca Alves Barros https://orcid.org/0000-0002-6662-5789

https://orcid.org/0000-0003-1018-5603

https://orcid.org/0000-0002-3080-0688

https://orcid.org/0000-0002-9035-486X 